\title{
Utilización de Plickers como plataforma didáctica para la evaluación del desempeño estudiantil en universidades
}

\author{
Carlos Vargas-Salgado ${ }^{a}$ Cristian Chiñas-Palacios $^{\mathrm{b}}$, Jesús Águila-León ${ }^{\mathfrak{c}}$ y Lina \\ Montuori $^{\text {d }}$
}

aDDepartament d'Enginyeria Elèctrica, Universitat Politècnica de València, carvarsa@upvnet.upv.es. ${ }^{\text {b,c }}$ Departamento de Estudios del Agua y la Energía, Universidad de Guadalajara, daniel.chinas@academicos.udg.mx)

${ }^{\mathrm{d}}$ Departamento de Termodinámica Aplicada, Universitat Politècnica de València, Camino de Vera, $\mathrm{s} / \mathrm{n}$, edificio 5J, 2 ${ }^{\mathrm{a}}$ planta. 46022 Valencia (España), 1montuori@upvnet.upv.es.

\begin{abstract}
The use of information technologies for the assistance of the teaching activity allows the teacher to be involved in the new teaching-learning trends. This work analyzes the benefits and advantages of using the Plickers tool as a digital platform for educational purposes to evaluate questionnaires, presenting a case study on the subject of Digital Electronics offered by the University of Guadalajara, Mexico, for the degree in Energy Engineering. For this purpose, a series of questions has been designed in Plickers, with the objective of evaluating Boolean algebra operations, together with their respective $Q R$ code response cards. The methodology that has been implemented is characterized by allowing an evaluation in real time and a gamification of the process as a prize is given to the student with the best score. Statistics on student performance have been made according to their score, by recurrence of score by question and by degree of difficulty per question, for the design of an adequate feedback. From the case study presented, the results shows that the group evaluated using Plickers has a regular performance, with $80 \%$ correct answers on average, the questions with the least number of correct answers are those involving more than 3 algebraic Boolean operations. It is concluded that, the Plickers tool is useful and efficient to achieve a dynamic and gamified evaluation process, where the teacher obtains the results of the evaluation in less time, as well as a set of data that allows a detailed analysis of the performance of his students, individually and groupally, for the design of strategies in order to correct the deficiencies detected in their students.
\end{abstract}

Keywords: Plickers, online tool, educational platform, learning evaluation, self-assesment.

\section{Resumen}

El uso de las tecnologías de la información en la actividad docente permite involucrar, además del profesor, al alumno en las nuevas tendencias de 
enseñanza-aprendizaje mediante la utilización de recursos tecnológicos. En este trabajo se analizan las ventajas de utilizar la herramienta Plickers como una plataforma digital con fines educativos para evaluar al estudiante, presentando un caso de estudio aplicado a la asignatura de Electrónica Digital del grado en Ingeniería en Energía ofertado por la Universidad de Guadalajara, México. Se han diseñado una serie de test en Plickers, con el objetivo de evaluar operaciones de álgebra booleana, junto con su respectiva hoja de respuestas con código QR. La metodología que se ha implementado se caracteriza por permitir una evaluación en tiempo real junto con una "gamificación" del proceso al dar un premio al estudiante con mayor puntaje. Se han realizado estadísticas sobre el desempeño estudiantil en función de sus aciertos, por recurrencia de acierto por pregunta y por grado de dificultad por pregunta, de tal manera que exista una retroalimentación adecuada. Del caso de estudio presentado, se obtiene como resultado que el grupo evaluado a través de Plickers tiene un buen desempeño, con un 80\% de aciertos en promedio, siendo las preguntas con menor número de aciertos aquellas que involucran más de 3 operaciones booleanas algebraicas (las que tienen mayor dificultad). Se concluye que la herramienta de Plickers es útil y eficaz para lograr una evaluación dinámica y gamificada, donde el profesor logra obtener en menor tiempo resultados de la evaluación, además de un conjunto de datos que permiten el análisis detallado del desempeño de los estudiantes, de manera individual y grupal, para el posterior diseño de estrategias que permitan subsanar las carencias detectadas.

Palabras clave: Plickers, herramienta online, plataforma educativa, evaluación del aprendizaje, autoevaluación.

\section{Introducción}

En muchas de las asignaturas cursadas hoy en día en una grado universitario no ser realiza prácticas de laboratorio. Esto puede ser debido al modelo de educación actual, a los costes o en algunos casos porque simplemente la enseñanza de asignatura no requiere experimentación, como es el caso de las asignaturas relacionadas con fórmulas y ecuaciones. Entre las asignaturas cuya enseñanza es principalmente teórica se encuentra álgebra: cálculo diferencial e integral y modelado matemático; mientras que otras deberían integrar una parte de teoría y una de experimentación como son las asignaturas de física, química y electrónica. Si el profesor planifica sus clases sin contemplar la experimentación y las herramientas didácticas adecuadas, el alumno puede perder el interés en la clase deviniendo en un pobre desempeño académico (Guzmán, 2019).

Para tratar de incrementar el interés por el aprendizaje de la materia de contendido tanto teórico como práctico, y con el objetivo de minimizar el número de estudiantes suspendidos para un mismo nivel de aprendizaje, sería útil para el profesor conocer información sobre el desempeño académico de cada alumno. Para ello, el uso de las tecnologías de la información y comunicación (TICs), en ambientes académicos ha crecido enormemente debido a la utilización de recursos tecnológicos para la docencia destinados a la innovación dentro y 
fuera del aula (Artal Servil, Peña Pellicer, Luesma Bartolomé, \& Gargallo Castel, 2018). Dentro de las TICs aplicadas a entornos educativos, la implementación del uso de los dispositivos móviles como material de trabajo en clase ha resultado de gran utilidad para el desarrollo profesional del alumnado (Lillo Giner, Camacho, \& Martínez Navarrete, 2018). Además, siguiendo una metodología de Flipped Learning, aplicada a cualquier rama del conocimiento, se consigue una mejora en el desempeño de los estudiantes (Escudero, Sanchez-vera, \& Muñoz, 2018).

Los entornos de aprendizaje virtuales aportan información precisa sobre el desempeño de los estudiantes. Un ejemplo de ellos es la herramienta Moodle. (Peña, 2018) realizó una análisis a los cuestionarios de evaluación de estudiantes de Ingeniería en Tecnologías Industriales de la Universidad de Zaragoza. Una metodología para la evaluación del aprendizaje enfocado al estudiante se muestra en (Reyes Tolosa \& Sahuquillo Navarro, 2018), donde se propone implementar una herramienta digital llamada Socrative, en donde por medio de formularios con cuatro posibles respuestas a modo de juego, los estudiantes van contestando cada una de las preguntas planteadas. Los estudiantes obtuvieron unos resultados satisfactorios al aplicar esta metodología de aprendizaje.

Por otro lado, el uso de juegos y competencias entre los estudiantes, conocido como gamificación o ludificación, desarrolla su nivel cognitivo, fortaleciendo los conocimientos sobre temas específicos (Sola Guirado, Castro García, \& González Sánchez, 2018). Una herramienta digital que incorpora dicha metodología de juegos se llama Kahoot! Se han realizado varios trabajos relacionados con el uso de dicha herramienta en diferentes áreas de conocimiento. Un ejemplo de ello es su uso en clases de estadística (Alcover Arandiga, Calduch Llosa, \& Vidal, 2018), medicina (González Peña, Jiménez Pérez, Salvador Palmer, Priego Quesada, \& Cebrían Ortíz, 2018), sistemas de información geográfica (Licorish, Owen, Daniel, \& George, 2018) y nutrición (Sempere Ferré, 2018), entre muchas otras áreas (Bicen \& Kocakoyun, 2018).

Actualmente existen muchas herramientas online que permiten la gamificación mediante cuestionarios hechos a través de Internet para su aplicación por medio de los dispositivos móviles. La Tabla 1 muestra una recopilación de algunas plataformas útiles para desarrollar las actividades académicas dentro del aula, ya sea para la realización de exámenes, la administración de las asignaturas por medio de apps que van almacenando la información sobre la materia cursada o para impartir clases virtuales con ayuda de software especializado para la realización de videoconferencias.

Plickers es una aplicación que involucra el uso de herramientas virtuales de carácter didáctico. Diversos trabajos presentados en publicaciones científicas, explican que esta plataforma tecnológica posee los atributos de un sistema de recolección de datos que se analiza en tiempo real, mostrando en la página web, después de cada acto de evaluación, la información del desempeño de los estudiantes (Mull, Howell, \& Tompkins, 2018). Esta plataforma permite incrementar la motivación del aprendizaje, además de ser una herramienta formativa con retroalimentación (Elmahdi, Abdulghani, \& Fawzi, 2018; Grison \& Ph, 2018; Silva, Sales, \& Castro, 2018).

Tabla 1. Herramientas TIC para beneficios del alumnado a través de apps gratuitas. 


\begin{tabular}{ccc}
\hline $\begin{array}{c}\text { Apps para } \\
\text { cuestionarios en } \\
\text { línea }\end{array}$ & $\begin{array}{c}\text { Apps para la } \\
\text { administración } \\
\text { del curso }\end{array}$ & $\begin{array}{c}\text { Apps para } \\
\text { videoconferencias }\end{array}$ \\
\hline Brainscape & Google Drive & Skype \\
Cerebriti Quizizz & Microsoft & Zoom \\
OneDrive & \\
Google Forms & Moodle & Google Hangouts \\
Infuse Learning & Paradiso & GoToMeeting \\
Kahoot! & eFront & Webex \\
Knowre & CourseSites & Rabbit \\
Plickers & DigitalChalk & JoinMe \\
ZipGrade & Sakai & \\
QuestionPress & Chamilo & \\
Socrative & & \\
\hline
\end{tabular}

\section{Objetivos}

El objetivo general de este trabajo es la integración de la plataforma virtual Plickers como Recurso tecnológico para la docencia y herramienta TIC, para la evaluación del desempeño de los alumnos en la asignatura de Electrónica Digital, de tal forma que los alumnos puedan autoevaluarse, mientras reciben retroalimentación por parte del profesor.

El objetivo general puede dividirse en los siguientes objetivos específicos:

- Conocer las ventajas y aprender a aplicar la plataforma virtual Plickers, de tal modo que el profesor pueda obtener en tiempo real resultados e información relevante del alumnado.

- Promover la migración de la información de las evaluaciones, estadísticas del grupo y desempeño de cada uno de los estudiantes, a un formato digital, a través de recursos pedagógicos en línea.

- Fomentar habilidades y destrezas en las tecnologías de información y comunicación para un correcto desarrollo en el perfil profesional del alumnado.

\section{Desarrollo de la innovación}

Para la integración de Plickers en la evaluación del alumnado, se ha seleccionado un grupo de 13 estudiantes de la asignatura Electrónica Digital, la cual se evalúa tradicionalmente mediante dos exámenes parciales y de test realizados mensualmente. Se ha introducido la plataforma Pickers en la realización de los test, que se llevarán a cabo la segunda semana de cada mes. Mediante esta herramienta el profesor diseña el cuestionario y el sistema de evaluación. Este trabajo presenta el diseño, la aplicación y los resultados obtenidos de haber utilizado Plickers. Los temas evaluados se muestran en la Tabla 2.

Tabla 2. Contenidos del quiz en Plickers

$\frac{\text { Electrónica Digital: Quiz \#1 Aritmética Digital }}{\text { Negación lógica }}$

(c) EY-NC-ND Universitat Politècnica de València de València 


\begin{tabular}{c}
\hline Suma lógica \\
Multiplicación lógica \\
Operaciones booleanas combinadas \\
Teoremas booleanos \\
\hline
\end{tabular}

La metodología empieza creando una cuenta gratuita desde el sitio web de https://get.plickers.com/, posteriormente se da de alta a varios grupos de clase, donde se añaden a los estudiantes y se crean las preguntas. Una vez registrada la clase y los alumnos, se procede a crear un nuevo set de preguntas por medio de la interfaz interactiva, donde previamente se selecciona la respuesta correcta. La Figura 1 muestra un esquema de la metodología implementada para la integración de Plickers para el diseño, aplicación y evaluación de test rápidos a lo largo del cuatrimestre, de acuerdo a una programación semanal.

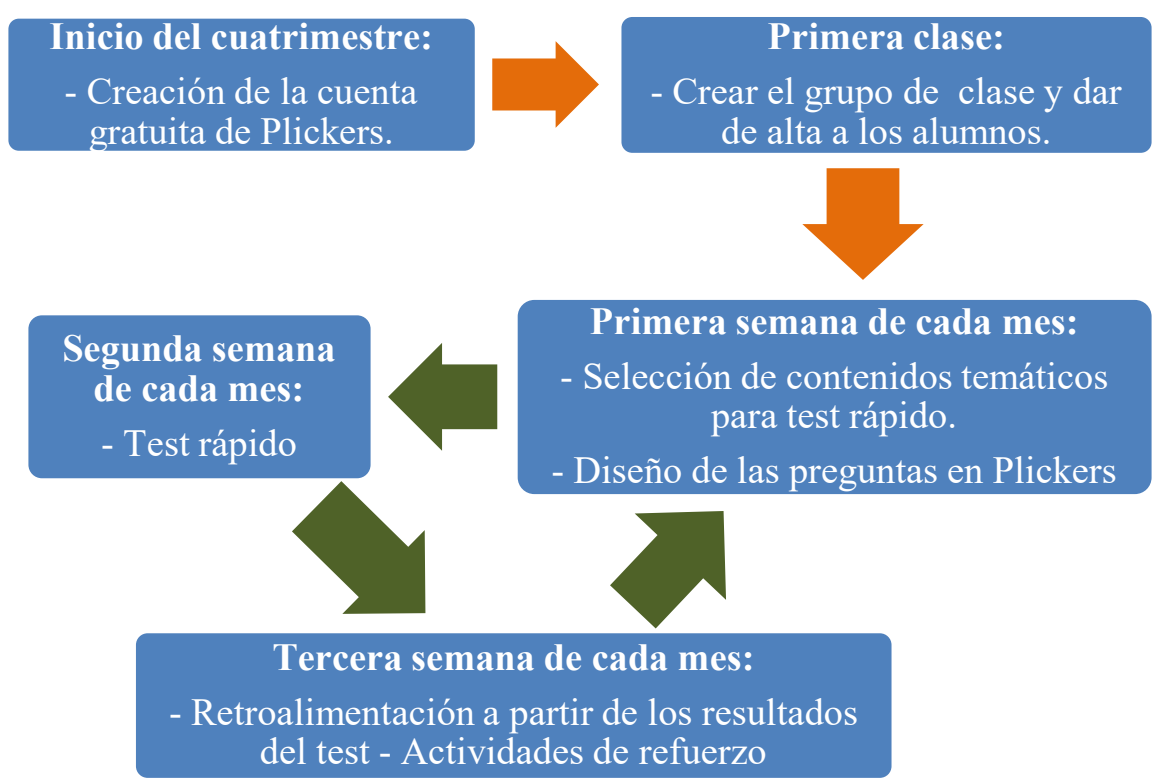

Fig. 1 Metodología de la implementación de Plickers para desarrollo y aplicación de test en clase.

La Fig. 2 muestra la ventana donde se diseñan las preguntas para el examen de la asignatura de Electrónica Digital, introduciendo el texto correspondiente para cada pregunta y seleccionando la respuesta correcta. Una vez diseñadas las preguntas, se procede a imprimir las hojas de respuesta (una por estudiante), que contienen un código QR impreso, (figura 3 d), que permite al profesor escanear la respuesta seleccionada por los alumnos a través del móvil con la app previamente instalada y mediante las credenciales que solo conoce el profesor, evaluando y almacenándose la puntuación para cada estudiante de manera automática en la base de datos de Plickers. 


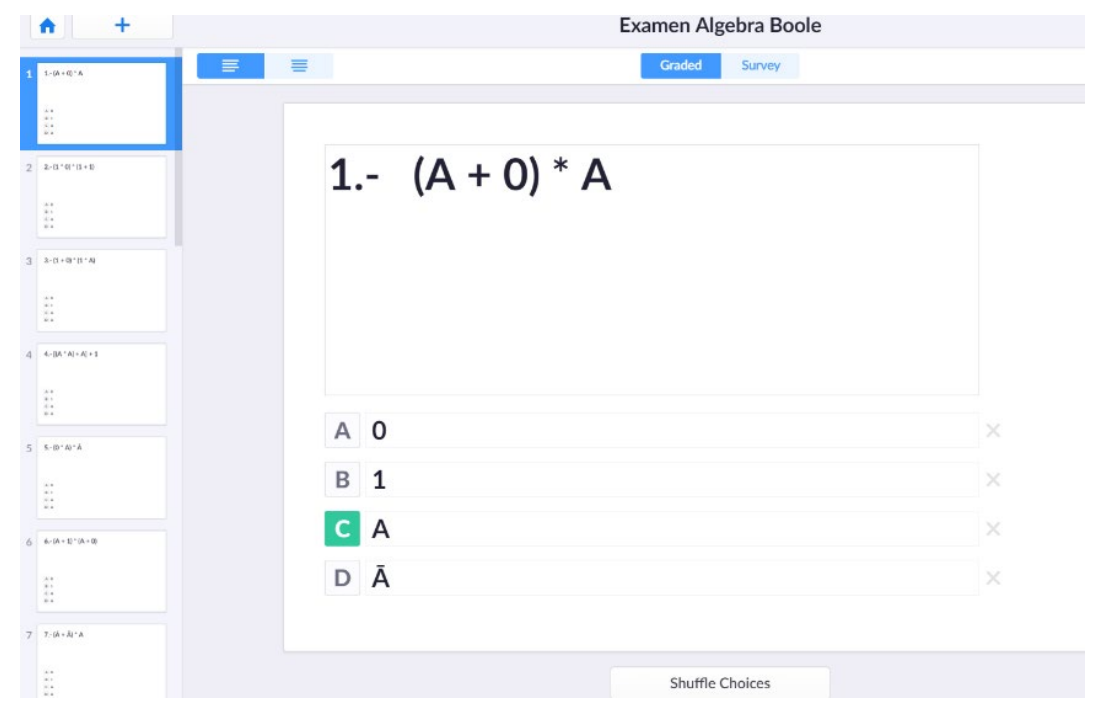

La Fig. 3 muestra el proceso para la realización de un test de 25 preguntas utilizando Plickers. La aplicación modifica de forma aleatoria el orden de las preguntas y de las respuestas para evitar el fraude. En la Fig. 3a se muestra al grupo de alumnos mientras se les explicaba las reglas del test que se explican a continuación:

1. Se reparten las tarjetas con el código QR a cada alumno. El primer estudiante de la lista de asistencia tendrá un folio con el número uno, el segundo tendrá el número dos, el tercero el número tres y así sucesivamente (figura $3 \mathrm{~d}$ )).

2. El folio con el código QR entregado a cada estudiante cuenta con las opciones; $a, b$, c y d, ubicadas en cada extremo (Figura 3d)). Para indicar la respuesta que consideren correcta, cuando el profesor lo indique, los alumnos enseñarán el folio con su respuesta ( $a, b, c$ o d) en la parte superior, visto desde el plano del profesor.

3. El estudiante dispondrá de dos minutos para pensar, analizar y dar la respuesta.

Una vez explicada la metodología, se procede al inicio del test. Las preguntas son visualizadas mediante el proyector. La Fig. 3b) muestra a los estudiantes en el proceso de selección de sus respuestas, mientras en la Figura 3c) se ve como el alumno indica la respuesta por medio del folio con el código QR. Un ejemplo de las primeras 3 tarjetas de respuesta entregadas a los estudiantes se muestra en la Figura 3d.

En la Fig. 4 se muestran los resultados de las preguntas 1 y 10 del test. En la Fig. 4a se muestra la pregunta 1, con el nombre de los estudiantes que realizaron la evaluación y el número de folio correspondiente. Las opciones vienen marcadas con los incisos de las letras a hasta la d, junto con la pregunta de la operación del Álgebra de Boole. En la Fig. 4b se muestra la pregunta número 10, con las posibles respuestas para dicha pregunta. La Fig. 4c indica la respuesta correcta para la operación algebraica de la pregunta 10. Finalmente, la Fig. $4 \mathrm{~d}$ muestra el resultado de la pregunta anterior y el número de alumnos que acertaron. En caso de la fig. $4 \mathrm{~d}$ se muestra en pantalla que el $100 \%$ de los estudiantes contestó correctamente esa pregunta. 
Fig. 2 Diseño de las preguntas de test en Plickers.

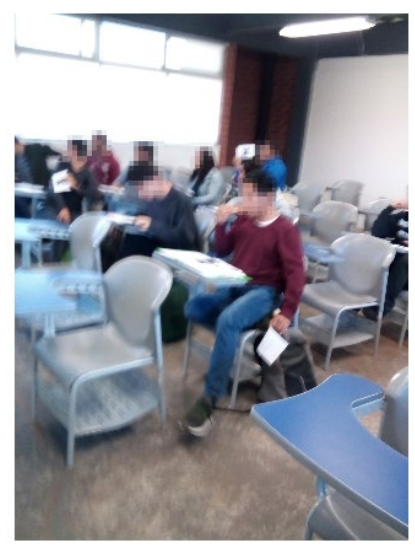

a)

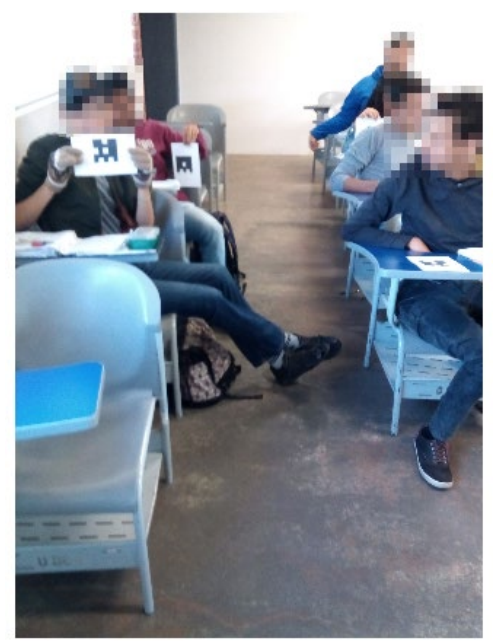

c)

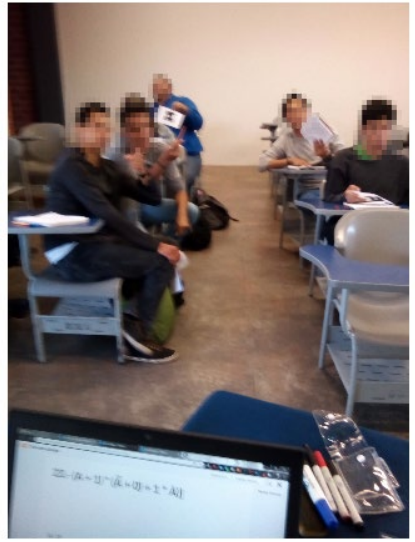

b)

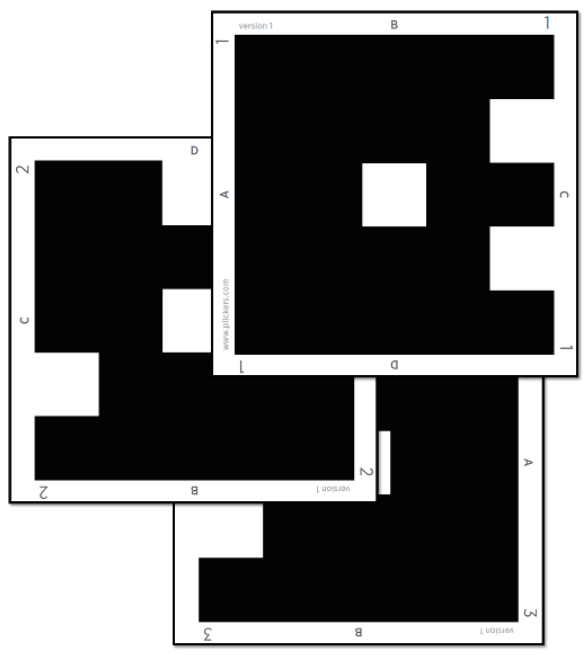

d)

Fig. 3 Proceso de aplicación de la herramienta Plickers para la evaluación de la asignatura Electrónica Digital. a) Se explica la metodología. b) Se da tiempo al estudiante para responder c) El estudiante indica la respuesta a través de la tarjeta. d) Tarjetas utilizadas.

$\mathrm{Al}$ integrar esta plataforma se aplica a los test rápidos el concepto de gamificación, con el objetivo de lograr que la motivación del estudiante. Quien logra el mejor resultado recibe como premio una caja de chocolates. Durante la realización del test se observó que el grupo estuvo motivado. Además, se preguntó a los estudiantes acerca de la evaluación, y los estudiantes manifestaron de forma general que les pareció innovador y divertido. 


$$
\text { 10. }-(1 * 1)+(\bar{A} * \bar{A})
$$

$$
\text { B } 1
$$

C. $A$

D $\bar{A}$

d)

Fig. 4 Implementación de las preguntas en Plickers.

\section{Resultados}

Se ha utilizado la proforma Plikers para evaluar a los estudiantes de la asignatura de Electrónica Digital. Al utilizar dicha plataforma, el profesor digitaliza por medio de la cámara de su móvil la respuesta de los estudiantes, los resultados se guardan automáticamente en una base de datos accesible desde el portal de Plickers o desde la aplicación del móvil. El alumno puede obtener los resultados de forma inmediata y el profesor ahorra tiempo de evaluación, además de obtener información estadística de los resultados (Fig. 5).

Debido a la versatilidad de Plickers, a la metodología utilizada en este trabajo se puede aplicar a otras asignaturas de grado o master para evaluar cualquier tipo de tema. A continuación, se realiza un análisis de los resultados obtenidos.

En la tabla 3 se observa y el número de aciertos la puntuación obtenida por cada estudiante. Los alumnos 2, 7 y 8 no se presentaron al examen, por lo no que se consideran para el análisis estadístico de los datos. 


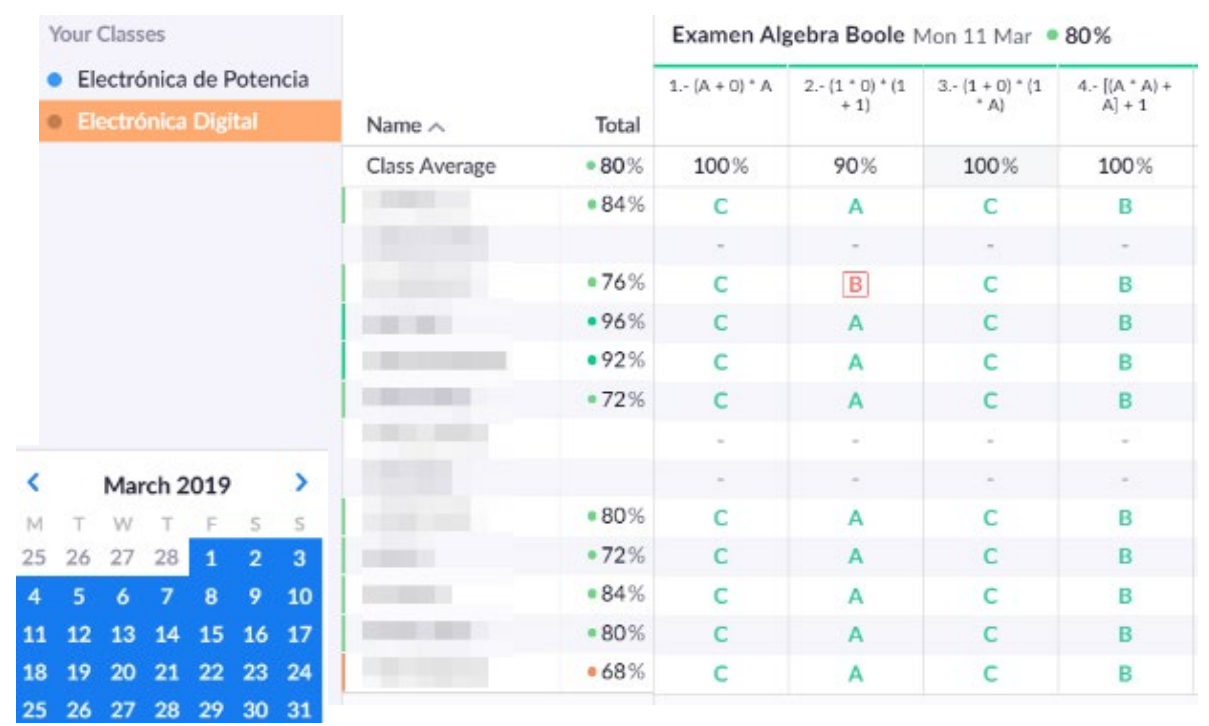

Fig. 5 Captura de pantalla de resultados que entrega Plickers

Tabla 3. Puntuación y número de aciertos para cada estudiante evaluado mediante Plickers

\begin{tabular}{ccc}
\hline Número de Alumno & Marcador & Número de aciertos \\
\hline 1 & $84 \%$ & 21 \\
2 & Ausente & Ausente \\
3 & $76 \%$ & 19 \\
4 & $96 \%$ & 24 \\
5 & $92 \%$ & 23 \\
6 & $72 \%$ & 18 \\
7 & Ausente & Ausente \\
8 & Ausente & Ausente \\
9 & $80 \%$ & 20 \\
10 & $72 \%$ & 18 \\
11 & $84 \%$ & 21 \\
12 & $80 \%$ & 20 \\
13 & $68 \%$ & 17 \\
\hline
\end{tabular}

En la figura 6 se muestra la gráfica obtenida a partir de la tabla 3, excluyendo a los alumnos no presentados al test. Con los resultados obtenidos de Plickers, es posible hacer de forma rápida un análisis detallado de las respuestas, como se muestra en la Figura 7. Esto permite identificar qué preguntas son más difíciles, y en cuál o cuáles tuvieron más aciertos o fallos, por lo que Plickers es también una herramienta estadística para que el profesor determine qué estrategias seguir para reforzar los conocimientos de sus alumnos, principalmente en los temas en los cuales la nota promedio obtenida fue baja. 
Utilización de Plickers como plataforma didáctica para la evaluación del desempeño estudiantil en universidades

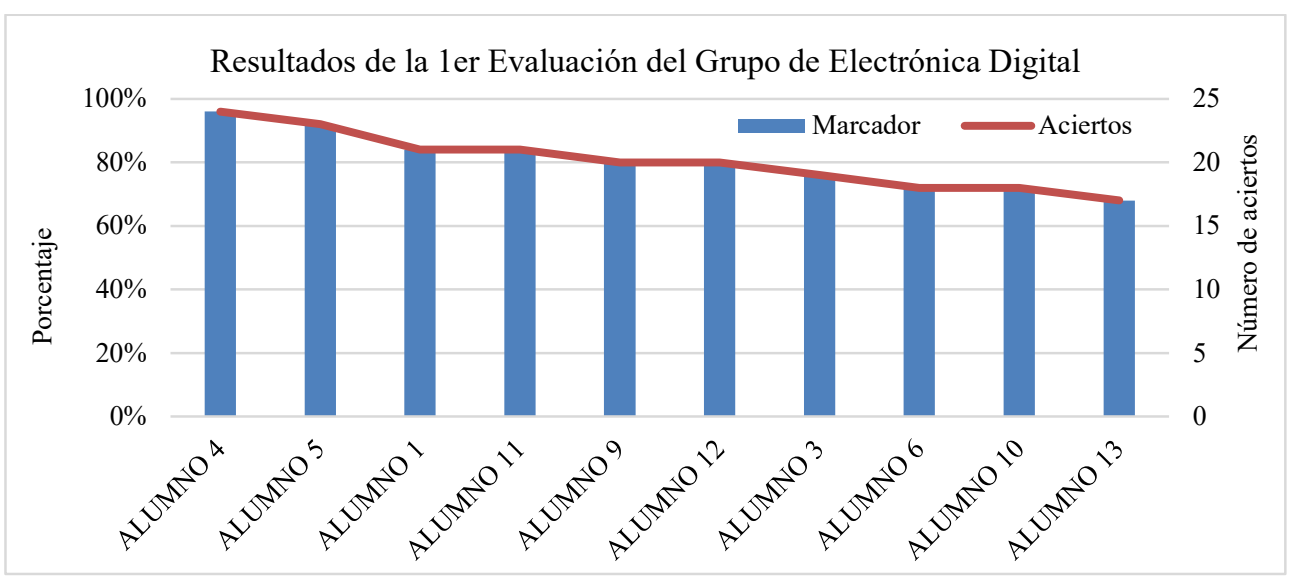

Fig. 6 Puntuación y número de aciertos de cada estudiante

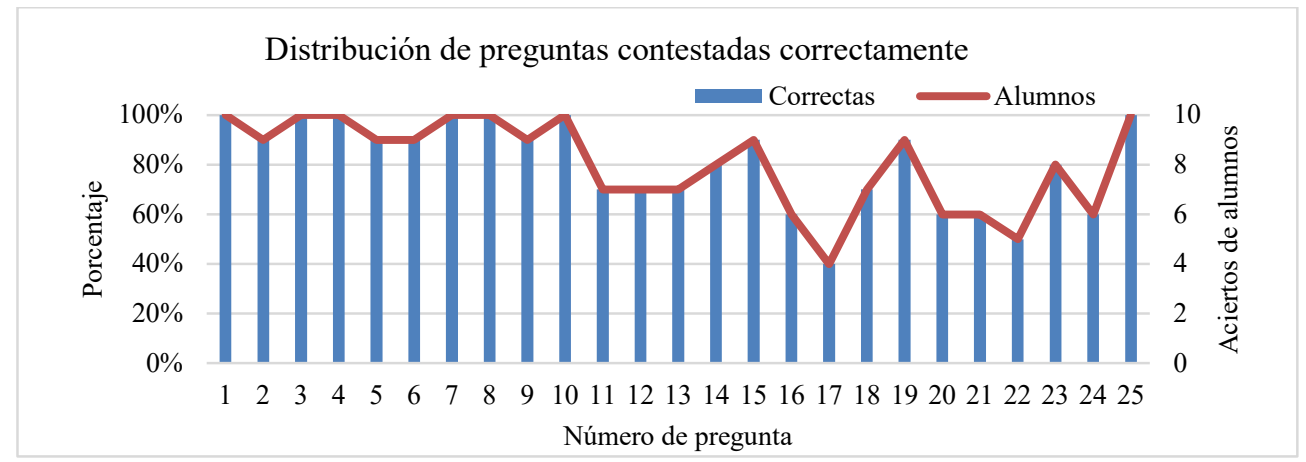

Fig. 7 Número de aciertos por cada pregunta del quiz.

Distribución ordenada del número de aciertos por pregunta del quiz

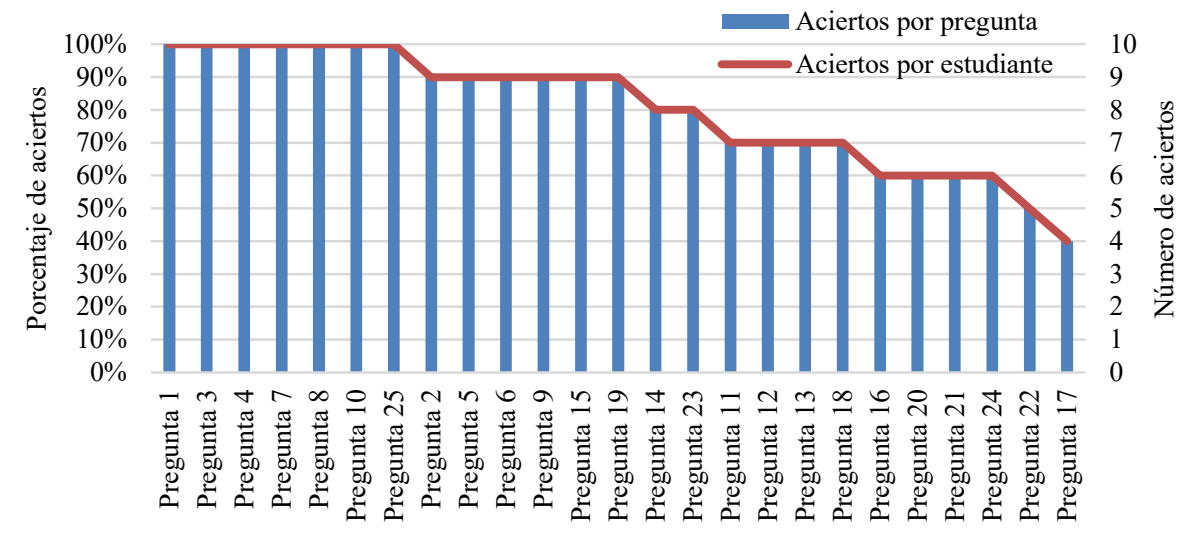

Fig. 8 Distribución de aciertos por pregunta

Las preguntas con mayor número de aciertos fueron la 1, 2, 3, 7, 8,10 y 25 (Figura 7), mientras que las de menor cantidad de aciertos fueron las preguntas número 16, 20, 21, 24, 22 y 17 (Figura 8). Considerando esta información, es posible establecer un orden de 
prioridad de temas a reforzar para subsanar las carencias presentadas por los estudiantes, resultando la lista de temas ordenada mostrada en la Tabla 5.

Tabla 5. Preguntas con menor número de aciertos y estrategias para mejorar desempeño

\begin{tabular}{|c|c|c|c|}
\hline $\begin{array}{l}\text { Número de } \\
\text { ejercicio }\end{array}$ & Ejercicio & $\begin{array}{l}\text { Porcentaje de } \\
\text { aprobación }\end{array}$ & Estrategia para mejorar \\
\hline 16 & $(0+\AA) * \bar{A}$ & $60 \%$ & Reforzar concomientos del Álgebra de Boole \\
\hline 20 & $(\tilde{\mathrm{A}}+0)+(\overline{\mathrm{A}}+\tilde{\mathrm{A}})$ & $60 \%$ & Repasar ejercicios de Álgebra de Boole \\
\hline 21 & $(\overline{\mathrm{A}}+1+\mathrm{A}) *(\overline{\mathrm{A}} * \mathrm{~A}+\overline{\mathrm{A}})$ & $60 \%$ & $\begin{array}{c}\text { Analizar detalladamente varios ejercicios } \\
\text { previos del Álgebra de Boole }\end{array}$ \\
\hline 24 & $\tilde{\mathrm{A}} *[(\tilde{\mathrm{A}}+1)+\overline{\mathrm{A}} *(0 * \overline{\mathrm{A}})]$ & $60 \%$ & $\begin{array}{c}\text { Observar cuidadosamente cada operación } \\
\text { Booleana }\end{array}$ \\
\hline 22 & $1 *[(\hat{\mathrm{A}}+1)+0 *(\mathrm{~A} * \overline{\mathrm{A}})]$ & $50 \%$ & $\begin{array}{l}\text { Identificar cada uno de las teoremas } \\
\text { Booleanos y su aplicación }\end{array}$ \\
\hline 17 & $\begin{array}{c}{[(\tilde{\mathrm{A}} * 1+\overline{\mathrm{A}})+(0 * \overline{\mathrm{A}}+\mathrm{A})]+[(\tilde{\mathrm{A}}} \\
* \overline{\mathrm{A}})+(\overline{\mathrm{A}}+\mathrm{A})]\end{array}$ & $40 \%$ & $\begin{array}{c}\text { Interpretar la lógica Booleana de acuerdo con } \\
\text { los teoremas aritméticos }\end{array}$ \\
\hline
\end{tabular}

\section{Conclusiones}

Plikers es una herramienta que utiliza los recursos tecnológicos para la docencia para evaluar a los estudiantes de una asignatura. Son varias ventajas detectadas al aplicar las metodologías de enseñanza/aprendizaje utilizando esta plataforma virtual:

- Rapidez a la hora de evaluar exámenes.

- Facilidad en la organización de las notas.

- Análisis estadístico del desempeño académico del alumnado.

- Identificación de las preguntas con más aciertos y más fallos y, por tanto, establecimiento de estrategias de enseñanza y aprendizaje a fin de reforzar las áreas con mayor dificultad.

- Feedback inmediato a los estudiantes.

En cuanto al análisis estadístico, Plikers calcula los valores promedio, máximo, mínimo y la desviación estándar a partir de la puntuación obtenida por los alumnos. Para la muestra analizada, se observa cómo la puntuación promedio fue de 80 puntos sobre 100 , mientras que la desviación estándar fue de un $8 \%$.

El análisis de los resultados muestra que la pregunta 17 fue la que tuvo mayor dificultad para a los estudiantes con un $40 \%$ de aciertos. El alumno número 4 tuvo obtuvo la mejor nota con un marcador de $96 \%$ de aciertos, y el alumno número 13 la de menor resultado, con un $68 \%$ de aciertos.

Finalmente, debido a su versatilidad, la herramienta Plikers se puede aplicar a otras asignaturas, en otros contextos y en cualquier programa universitario de grado o master. 
Utilización de Plickers como plataforma didáctica para la evaluación del desempeño estudiantil en universidades

\section{Referencias}

AlCOVER ARANDiGA, R. M., CALDUCH LlOSA, Á., y VIDAL, S. (2018). "Nos divertimos y aprendemos con Kahoot! en las clases de Estadística". Congreso Nacional de Innovación Educativa $y$ de Docencia En Red, 165-175. $<$ https://doi.org/10.4995/inred2018.2018.8642> [Consulta: 9 de marzo de 2019]

ARTAL SERVIL, J. S., PEÑA PELLICER, B., LUESMA BARTOLOMÉ, M. J., y GARGALLO CASTEL, A. F. (2018). "Herramientas TIC y software específico. Un buen complemento en el aula universitaria". Congreso Nacional de Innovación Educativa y de Docencia En Red, 324-338. <https://doi.org/10.4995/inred2018.2018.8750> [Consulta: 9 de marzo de 2019]

BICEN, H., y KOCAKOYUN, S. (2018). "Perceptions of Students for Gamification Approach: Kahoot as a Case Study". International Journal of Emerging Technologies in Learning (IJET), 13(02), 72-93. Retrieved from <http://online-journals.org/index.php/ijet/article/view/7467/4807> [Consulta: 9 de marzo de 2019]

ELMAHDI, I., ABDULGHANI, A.-H., y FAWZI, H. (2018). "Using Technology for Formative Assessment to Improve Students' Learning". TOJET: The Turkish Online Journal of Educational Technology, 17(2), 182-188. [Consulta: 9 de marzo de 2019]

ESCUDERO, E., SANCHEZ-VERA, I., y MUÑOZ, U. (2018). "Análisis de la metodología Flipped learning en el entorno de la práctica de la Fisiología Médica". Congreso Nacional de Innovación Educativa $y$ de Docencia En Red, 651-662. $<$ https://doi.org/10.4995/inred2018.2018.8616> [Consulta: 9 de marzo de 2019]

GONZÁLEZ PEÑA, R., JIMÉNEZ PÉREZ, I., SALVADOR PALMER, R., PRIEGO QUESADA, J. I., y CEBRÍAN ORTÍZ, R. M. (2018). "Aplicación del KAHOOT para la mejora de la adquisición de los objetivos del aprendizaje en el alumnado de Fisiología de los órganos del lenguaje y la audición", 230-237. $<$ https://doi.org/10.4995/inred2018.2018.8728> [Consulta: 9 de marzo de 2019]

GRISON, S. (2018). "Teaching Toolbox Series : Using " Plickers " to Increase Engagement and Learning What are our goals today?" [Consulta: 9 de marzo de 2019]

GUZMÁN, M. (2019). "La gestión académica en el nivel superior frente a los desafíos de la formación investigativa de los estudiantes". Revista Multidisciplinaria de Avances de Investigación, 5(1), 1-15. [Consulta: 9 de marzo de 2019]

LICORISH, S. A., OWEN, H. E., DANIEL, B., y GEORGE, J. L. (2018). "Student perception Kahoot". Research and Practice in Technology Enhanced Learning, 13(9), 1-24. $<$ https://doi.org/10.1186/s41039-018-0078-8> [Consulta: 9 de marzo de 2019]

LILLO GINER, S., CAMACHO, M. D. M., y MARTÍNEZ NAVARRETE, N. (2018). "Uso de dispositivos móviles para el desarrollo de las prácticas de laboratorio utilizando una herramienta online". Congreso Nacional de Innovación Educativa y de Docencia En Red, 238-249. <https://doi.org/10.4995/inred2018.2018.8889> [Consulta: 9 de marzo de 2019]

MULL, C., HOWELL, N., y TOMPKINS, C. (2018). "Plickers for Success : A Technological Tool for Advancement in Data Collection". Journal of Extension, 56(7), 1-4. [Consulta: 9 de marzo de 2019]

PEÑA, B. (2018). "Uso de recursos y rendimiento en las actividades evaluación: análisis y comparación de resultados en el Grado en Ingeniería de Tecnologías Industriales". Congreso Nacional de Innovación Educativa $y$ de Docencia En Red, 23-34. $<$ https://doi.org/10.4995/inred2018.2018.8583> [Consulta: 9 de marzo de 2019] 
REYES TOLOSA, M. D., y SAHUQUILlO NAVARRO, O. (2018). "Propuesta de una metodología de evaluación del aprendizaje basada en las TIC". Congreso Nacional de Innovación Educativa y de Docencia En Red, 691-701. $<$ https://doi.org/10.4995/inred2018.2018.8622> [Consulta: 9 de marzo de 2019]

SEMPERE FERRÉ, F. (2018). "Kahoot como herramienta de autoevaluación en la universidad". Congreso Nacional de Innovación Educativa y de Docencia En Red, 250-255. $<$ https://doi.org/10.4995/inred2018.2018.8730> [Consulta: 9 de marzo de 2019]

SILVA, D., SALES, G., y CASTRO, J. (2018). "A Utilização Do Aplicativo Plickers Como Ferramenta Na Implementação Da Metodologia Peer Instruction". Revista Eletrônica Cientifica Ensino Interdisciplinar, 4(12), 502-516. $<$ https://doi.org/10.21920/recei72018412502516> [Consulta: 9 de marzo de 2019]

SOLA GUIRADO, R., CASTRO GARCÍA, S., y GONZÁlEZ SÁNCHEZ, E. (2018). "Influencia de la gamificación en diferentes cursos y tipos de alumno". Congreso Nacional de Innovación Educativa $y$ de Docencia En Red, 72-80. $<$ https://doi.org/10.4995/inred2018.2018.8598> [Consulta: 9 de marzo de 2019] 